\title{
Urgences
}

\section{" Dieu, un corps brûlant dans la neige fondante des religions "}

\section{Dominique Garand}

Numéro 23, avril 1989

Lisière du livre

URI : https://id.erudit.org/iderudit/025519ar

DOI : https://doi.org/10.7202/025519ar

Aller au sommaire du numéro

Éditeur(s)

Urgences

ISSN

0226-9554 (imprimé)

1927-3924 (numérique)

Découvrir la revue

Citer cet article

Garand, D. (1989). " Dieu, un corps brûlant dans la neige fondante des religions

". Urgences, (23), 77-88. https://doi.org/10.7202/025519ar d'utilisation que vous pouvez consulter en ligne.

https://apropos.erudit.org/fr/usagers/politique-dutilisation/ 


\section{DOMINIQUE GARAND}

\section{«DIEU, un corps brûlant dans la neige fondante des religions»}

L'oeuvre de Carole Massé me fait penser à celle d'Olivier Messiaen: elle s'attaque à des thèmes impossibles et abstraits que même la philosophie n'ose plus aborder. Dieu, L'existence, Nobody, L'autre, Hommes, tels sont les principaux jalons de ce parcours poursuivi solitairement avec une constance et un entêtement qui surprennent, peut-être même agacent. Mais tout comme chez Messiaen, l'apparente lourdeur répétitive et la difficulté certaine du phrasé conduisent l'écriture à des déflagrations inouïes, presque assourdissantes d'intensité: on a la sensation qu'il s'est passé quelque chose, qu'un saut s'est opéré dans les mots. L'entreprise de Carole Massé est une véritable aventure de la subjectivité. I I faudra reconnaître un jour que seule une telle subjectivité, affirmée à travers les obstacles et écrans (et l'auteure a choisi d'emblée le plus mystifiant: Dieu!), puisse être ouverture à une réelle communication, étant seul gage d'une Parole prononcée. Parole de Carole...

Cette parole exigeante et de plus en plus joueuse, issue à l'évidence de l'analyse, visite en premier lieu la différence sexuelle et le rapport du corps à la voix, en passant par la mémoire et le Nom. Peu d'oeuvres, à ma connaissance, questionnent avec autant d'acharnement le lien social, tel qu'il s'avoue dans le langage et dont la métaphore, ou plus carrément le fondement, se trouve dans le lien familial incestueux. Si cette oeuvre tarde à être vraiment lue, il faut sans doute l'expliquer par le tabou tenace sur cette question, entretenu tout autant par ce qu'on appelait naguère le phallocentrisme, que par le féminisme dogmatique (gynocentrique).

Paru en 1979, Dieu est le premier volet de ce que Carole Massé, après L'existence et Nobody, considérera comme son «roman familial». Pour parler adéquatement de ce roman, il faudrait exposer sa structure poético-narrative très complexe, son énonciation à plusieurs voix, sa pluri-signifiance qui défie toute réduction au signe. Cette analyse, bien qu'inédite, a déjà été menée; je m'en tiendrai toutefois, pour respecter la problématique théorique de ce numéro d'Urgences, aux grandes lignes générales, privilégiant l'examen des politiques titrologique et éditoriale. 


\section{A. Ce qui s'annonce d'un couvert à l'autre}

Un titre percutant, provocateur: Dieu. Le seul précédent de la langue française que je connaisse est le titre de Victor Hugo, un Hugo on ne peut plus plongé dans la "dix-neuviémité» (voir Philippe Muray): occultisme et socialisme mêlés. Bref, un peu ronflant, totalisateur, en mal de faire aller l'histoire dans le bon sens. Au milieu des années 70, Philippe Sollers commence Paradis et réactive avec quelques autres la prise en charge littéraire de la théologie. Le projet de Carole Massé n'est pas étranger à ce mouvement d'investigation du refoulé, mais pour elle le défi comporte une autre dimension: comment penser le sujet féminin à travers cet héritage? De quelle manière parlera-t-il, ce sujet? Réussira-t-il premièrement à parler, à jouir de sa parole? Enfin, comment le féminisme se débrouillera-t-il avec la psychanalyse et la religion issue de la Bible, toutes deux ancrées au Nom du Père?

Le nom de Dieu, en bleu, domine la page de couverture dont il occupe les deux-cinquièmes. Deux autres cinquièmes sont couverts par l'illustration «La Sainte Vierge corrigeant l'enfant Jésus sous les yeux de 3 témoins: André Breton, Paul Éluard et l'artiste" (Max Ernst, 1926). Cette illustration «renversante», iconoclaste, suffit à nous avertir qu'il ne s'agit pas d'un ouvrage pieux mais cela, déjà, la signature de l'éditeur (Les Herbes Rouges) nous avait permis de le deviner. II y a contrariété entre l'illustration et le titre, ou plutôt, il y a ironisation du titre par l'illustration: c'est bien Dieu enfant qui est battu par la Vierge-Mère: la Vierge porte l'auréole, celle de l'enfant Jésus est tombée par terre... On pressent qu'un mauvais sort est réservé à Dieu dans ce livre et on lira effectivement à la page 121: “une femme qui n'est plus mère ni vierge corrigera l'enfant-Dieu devant trois témoins: la soeur du frère et l'écrivain ici qui s'en souvient 's (est-il besoin de souligner la contradiction temporelle déterminante de la phrase: l'écrivain se souvient d'un fait qui aura lieu. Serait-il historien-prophète?). Toutefois, ce passage laisse croire également que le texte se détachera du programme narratif inscrit dans l'illustration. En effet, cette femme qui bat l'enfant est encore mère et, de plus, elle est sainte (l'auréole). Les voyeurs derrière sont des hommes, deux poètes et un peintre, on ne sait s'ils s'amusent de cette scène ou s'ils s'identifient douloureusement au bambin. Quoi qu'il en soit, le renversement ici est clair: l'enfant a beau être Dieu, il reste l'enfant de sa mère et celle-ci a tout pouvoir sur lui.

Je passe au nom de l'écrivain, Carole Massé. On ne l'aperçoit pas immédiatement puisqu'il est littéralement écrasé, à l'instar de 
l'inscription "roman", entre "Dieu» et l'illustration: entre le Père immense, infini (le bleu donne cette impression «céleste») et la Mère toute-puissante, voguent ensemble «Carole Massé» et son «roman». À moins que d'avoir ainsi posé son nom au-dessus de l'illustration veuille dire: «ci-dessous, Carole Massé battant l'enfant Jésus»; ou encore «ci-dessous, Carole Massé enfant, battue par sa mère la Vierge"? À la lecture du texte cette deuxième hypothèse s'avère signifiante, l'auteure tirant de son nom (qui est le nom de sa mère, c'est-à-dire du père de celle-ci) une série de verbes passifs qui en font la victime d'une agression répétée: «massée mâchée mâchurée macérée maillée mangée matricée mamelonnée mimée médusée mouillée morcelée" (p. 114; la suite du texte poursuit l'isotopie dans l'évocation d'une naissanceexpulsion, du lait qui monte, d'un contact-séparation).

J'ouvre le livre: "Dieu de Carole Massé est le vingtième ouvrage publié dans la collection lecture en vélocipède". Je donne un coup de pédale et trouve, page 2, le titre de l'illustration de couverture, son copyright et le nom des responsables de la couverture et de la mise en page, tous deux des ateliers «l'Enmieux». C'est encourageant, ça invite à continuer. Page 4: «Dieu», tout seul au milieu de la page blanche. Page 5, indication du premier livre de l'auteur: Rejet, poèmes, paru aux Éditions du Jour en 1975. L'auteur a vu le jour dans un mouvement de rejet. II y a dans ce titre l'indice d'une révolte et l'annonce d'un thème fondamental de Dieu: les «restes", les "pertes", l'évacuation quasi anale du corps maternel. La page de titre répète la couverture, sans illustration et sans l'indication «roman». La page 6 donne l'adresse de l'éditeur et du distributeur et inscrit Dieu (le livre) dans la légalité («Dépôt légal - premier trimestre 1979») comme dans l'espace québécois ("Bibliothèque nationale du Québec»). Ainsi, non seulement Dieu est-il battu mais il a trouvé une loi pour le soumettre!

Une photographie couvre presque toute la page 7 , nous montrant une enfant d'environ 7-8 ans à moitié cachée derrière un arbre. On ne lui voit pas les mains, ni la bouche. Elle porte des cache-oreilles et nous regarde gravement d'un oeil sombre. La psychanalyse nous suggère d'associer l'arbre au Phallus, hypothèse amplement soutenue par le texte. Ce texte, que le lecteur découvre ensuite, semble écrit d'un seul souffle, sans aucune division en chapitres ou en paragraphes. À vol d'oiseau, on dirait une tempête de mots jetés pêle-mêle, la ponctuation étant inexistante, ou à peu près. L'espace entre les mots varie. C'est dense, cela s'étend sur 114 pages. Quelques passages sont en italiques, des citations dont on nous indique les auteurs, page 126. À la page 
127, la photographie d'une bouche féminine: un trou noir entouré de deux lèvres deux fois plus grandes que nature. Je songe encore à Victor Hugo: «Ce que dit la bouche d'ombre...» Bien sûr, l'allusion sexuelle est immédiate. Mais que dit cette bouche? Aspire-t-elle? Souffle-t-elle? Veut-elle embrasser? Énonce-t-elle une autre Loi, comme pourrait le suggérer le prénom de la photographe, Lois? Mais cette bouche est trop seule, ouverte comme une plaie... Et le symbole de la Loi est plutôt un doigt pointé en l'air.

Enfin, au dos de la couverture, une grande photo bleuie nous montre une belle jeune femme (qui est Carole Massé): épaisse tignasse léonine, coiffée comme une petite fille avec des barrettes, gilet de laine dont le col entoure le cou jusqu'au menton. Les bras croisés (elle aussi cache ses mains), le regard est calme. L'envers de Dieu?

Peut-on avancer qu'une traversée se fait. Du nom de Dieu et de l'image de la Mère, par l'enfant sans voix cachée derrière l'arbre, on aboutit à une voix pure sans visage, la parole, la bouche d'ombre, qui est aussi métonymie du sexe féminin, et enfin à une femme qui nous «regarde du haut de son vingtième siècle». Cette femme est-elle celle qui, n'étant plus mère ni vierge, a corrigé l'enfant-Dieu? Cette femme, quelle est sa parole ou son nom dont la valeur marchande est fixée à $6,95 \$$ et qui, tout comme la petite fille à l'arbre, est photographiée par Serge Laurin? II va sans dire que le support matériel du livre ne peut donner pleine signification à tous les signes qu'il donne à déchiffrer et qu'il est nécessaire de passer par le texte.

\section{B. Le titre, béance du livre}

Une longue tradition romanesque considère le nom propre comme le titre par excellence: nom du héros ou de l'héroïne, du moins du personnage central, associé au nom du livre. Qu'en est-il de Dieu, Nom Propre parmi les noms propres? Dieu (du moins celui des judéo-chrétiens) a sa Parole, son Livre, mais comment peut-il être réduit à l'état de personnage, comment faire son procès?

Le titre (Dieu) est béance du livre, ce livre qui se veut «sans commencement ni fin». Le graphisme du titre (le «U» ressemble à un " $V »)$ nous dirige vers l'anagramme de Dieu qu'est le mot «vide». Texte sans clôture? Texte des limites? Sans ponctuation, nos chers repères habituels! Peut-être Dieu est-il un sujet tabou 
parce que justement coeur du vide. Mais qu'est-ce qui se trame sous ce nom de Dieu, comment l'entendre?

«Dieu est un mot» (p.25).

«God is a concept by which we measure our pain» (Lennon, cité p. 45 et 47 ).

Ce mot servirait donc de prétexte pour parler d'autre chose, pour écrire une pré-Face par exemple: «préface de l'impossible reflet l'insupportable Figure sans commencement ni fin" (p. 11). Faire le procès de Dieu, en explorer les soubassements est un risque périlleux, la voix risque de s'y engloutir: "confondra-t-elle le mensonge?», est-il demandé à quelques reprises (p. 11, 13, 16, 18). Car Dieu est posé ici comme une instance imaginaire, une Figure, une métaphore, assez vide pour que d'autres figures puissent se substituer à elle. Comment les repérer: le Père, tous les Pères de l'Église et de la Science, mais surtout celle dont on ne parle pas: la Mère?

On dit que Dieu qu'il est Un (indice de notre fragmentation) et qu'il est l'Autre (indice de notre manque). On sait aussi que voir Dieu c'est mourir. Mais quel est ce Dieu que l'on ne saurait voir? «je me souviens d'avoir ressenti un jour devant ma mère cette inquiétude fulgurante de me retrouver une seconde face à la Vérité et je me suis bouché les yeux et les oreilles" (p. 59). Mais qu'y a-t-il chez la mère qui fasse Vérité de manière aussi terrifiante? «Dieu est mère son sang coulant nous roucoule l'affre d'en découler l'offre d'y écouter des mouvements de corps y risquer une pensée" ( $p$. 53); «reconnois donq la tragedie n'a ù autre origine les mots qui courent à mêler la genealogie à faire Dieu la vraye mere quand elle n'est en amour avec son mari» (p. 54). Finalement, le noeud de l'affaire tourne autour de la reproduction, de cette dette éternelle envers l'espèce. Le texte présent tente une sortie hors de ce cercle, acceptant l'errance d'une écriture sans cesse déprise de la logique causale reproduite dans une syntaxe normative. Errance qui n'est pourtant pas insignifiance, mais au contraire sursignifiance, par le rythme, l'association libre. Dieu reconnaît également le lien de la reproduction et de la ponctuation; la procréation ne s'emmêle toujours qu'après le point, dans la censure du point, quand la parole n'en peut plus, quand le souffle vient à manquer et qu'il faut faire station: panne de courant, tout le monde au lit! $\mathrm{Au}$ contraire, le texte de Dieu court et court, bute à maints endroits, revient sur ses pas, ralentit, accélère, traverse lettre à lettre tout l'alphabet jusque dans la débâcle finale qui emporte tout, à perte de vue. 
Les passages où il est question de Dieu sont donc fondamentaux; le titre nous invite à les prendre en considération. Contre Dieu (au sens de «tout contre») s'élabore de façon insistante un concept du féminin qui n'est pas sans rappeler celui proposé par Baudrillard dans De la séduction: Dieu est du côté du plein, de la totalité, de l'image, de la représentation, du Pouvoir et de la Loi; le féminin est la faille dans l'ordre, la brèche qui fissure la représentation, le reste qui confond la totalisation. Pour Carole Massé, le féminin est de l'inimaginaire: «l'inimaginaire qu'ils pansent et compensent par le désir de Dieu être l'enfant avalé ou le désir d'être Dieu le Père avalant

l'inimaginaire que nous dépensons dilapidons malgré nous dans notre absence notre évacuation même-

l'inimaginaire où manque l'Un avec tous les risques d'aphasie de catalepsie de déraison» (p. 117). Le rapport entre la femme et la mère est ambigu: la mère serait le côté représentable de la féminité, une sorte de concession donnée à l'Homme (l'espèce) pour nourrir son propre imaginaire: "et voilà le fantôme féminin feignant l'homme feignant la femme pour feindre la mère seule féminité permise pour reproduire l'Homme et son fantôme féminin» (p. 79). Permise, c'est-à-dire mise par le père, seule divinité qui permet l'élaboration de l'humanité. Que comprendre de cette tactique de la feinte (ou de la fente, ou de la fiente)? "feindre la mère exclure la femme dès lors support de l'irreprésentable et qui interpelle d'ailleurs d'autre part du reste cette économie finie s'ordonnant depuis cette brèche cette faille cette fente» (p. 21-22). C'est ce qui est exclu, au moins, qui finit par déborder ce qui l'exclut: «ce que nous sommes toutes surcharges ratures biffures erreurs fautes maladresses dans la sainte écriture de la Loi» ( $p$. 102). Depuis cette exclusion le sujet-femme, celui qui jouit hors de la reproduction, devient menace pour la représentation: «femme en moins cicatrice d'où sourd au cours des âges la menace constante de son retour diable antéchrist chair matière désir défigurant la sainte Face» (p. 78). En effet, si «Dieu est une dictature fasciste» (p. 90), tout ce qui n'entre pas en lui l'excède et le menace: ce qu'il a exclu, il craint sans arrêt de le voir resurgir.

Le procès de Dieu (il serait préférable de dire que l'écriture de Massé descend en Dieu, comme Dante aux enfers) aboutit ici à un athéisme conséquent, informé des multiples formes que peut revêtir la religion. Athéisme qui, au contraire, de l'agnosticisme toujours un peu crispé et teinté de ressentiment, est à la fois contestation, révolte et affirmation, percée de jouissance. Le scandale est bien de démasquer la servitude qui sous-tend le désir de Dieu, du moins ce qu'il y a de problématique pour une femme dans ce rapport, mais aussi d'identifier Dieu à la Mère archaïque: Dieu est Mère, 
nostalgie de la Mère, avec tout ce que cela peut comprter de doux et d'étouffant à la fois. Sur ce point, Carole Masse se rapproche du YHWH hébreu, lui aussi irreprésentable, toujours opposé à sa réification et allié d'un peuple marginalisé, petit reste. Pour que l'irreprésentable surgisse, il faut constamment nier le trop représenté, il faut abolir le nom de Dieu, ou plutôt faire la critique constante de ce qu'il camoufle. Toutefois, l'auteure franchit un pas de plus en refusant également l'ordre patriarcal dans son instrumentation répressive. L'écriture de Carole Massé n'est pas une opposition frontale ou une négation de la Loi: il ne s'agit pas d'instaurer un autre ordre sous un autre Dieu, créer une autre religion, ni même l'inverse, dénier tout religieux et prétendre s'en passer. Le religieux est au contraire accepté, traversé, parlé. L'auteure a recours à des images superbes pour décrire ce processus: "l'écoutant impassible trouve sur le bord d'une enfance criante une femme imparlée dont le corps brûlant roule dans la neige fondante des religions et embrasse le risque d'une philosophie» (p. 105). Et enfin: "elle dit oui sans Dieu sans ascétisme sans mysticisme mais elle dit oui contre Dieu sans confusion sans équivoque» (p. 119120). Seule une parole rythmée peut permettre d'assumer un tel choix sans angoisse.

\section{Ceux qui sont cités à comparaître: voix des autres et émer- gence du sujet}

Tout ce qui précède resterait abstrait si je ne décrivais pas comment cela s'effectue dans Dieu. II faut aussi tenter de répondre aux questions posées par l'examen de la politique éditoriale. L'onomastique est la racine principale de la politique titrologique. L'analyse des noms propres est en outre encouragée par l'appareil éditorial qui prend la peine de nous indiquer, page 128, la provenance des citations disséminées dans le texte.

Le travail de la citation est en effet, en Dieu, considérable. Les citations surgissent dans le corps du texte et ne sont indiquées que par des italiques. Elles nous permettent de comprendre que Dieu n'est pas le "brainstorming" irréfléchi qu'une lecture superficielle peut laisser supposer. C'est au contraire un livre savant. Carole Massé a puisé chez des auteurs de cultures différentes et aussi éloignés dans le temps que Colette, Homère, Mao, John Lennon, Emily Dickinson, etc. S'ajoutent aux citations explicites des renvois implicites à de grands textes de l'histoire, aux mythes, à la Bible, à Victor Hugo, à Chateaubriand, à Nietzsche, etc. Des peintres, des cinéastes, des photographes sont aussi mentionnés 
comme adjuvants ou opposants. Finalement, on retrouve des noms de ville et quelques titres d'oeuvres littéraires ou picturales. Ce que la critique appelle intertextualité est donc visiblement agissant dans Dieu. De tels clins d'oell, avoués ou non, constituent une sorte de trame sonore où les voix des autres et de l'histoire font écho, mais modulées par le souffle du texte, c'est-à-dire par le je du discours qui s'accapare l'entier du langage, oeuvres et mots, signes et proverbes, et le transforme par sa pratique signifiante.

Règle générale, les citations sont accompagnées de dates, sauf les citations d'hommes (exception faite pour Homère, mais il y a un débat sur l'appartenance de son sexe). Des paroles d'hommes, sont reçues mais ne constituent pas les étapes essentielles de l'histoire du sujet féminin qui se forme dans ce livre. La dernière date mentionnée est 19781 .

Dieu, enseigne du grand Autre, fourmille de la parole de "petits autres» disséminés dans l'histoire. L'histoire du “je» se constitue, se trame dans la suite, par et au travers la voix des autres. On sait que l'un des idéaux les plus tenaces du romantisme est l'originalité du héros, lui qui prend figure de Dieu (ou de diable) au milieu des autres détestables. Ici, aucun héros, aucune héroïne: une voix qui porte plusieurs noms, mais d'abord la voix des autres, avec ce petit filet entre eux immiscé qui tente de se frayer une voie.

Ces autres ne constituent pas une généalogie: une écriture féministe s'est fondée une généalogie déjà, en remplaçant les figures paternelles par des figures féminines et maternelles. Dieu ne participe pas de cette entreprise: il n'y a pas, répétons-le, dénégation des Pères, il n'y a pas non plus mythologisation et sacralisation des Mères répertoriées dans l'histoire. Comme je le faisais remarquer dès le départ, sur l'illustration de la page frontispice, le sujet-femme, encore enfant sans bouche et sans voix, est posté à l'ombre du Phallus, qui est l'arbre (généalogique). Tout au long du livre resurgissent les voix des Pères, les voix du Pouvoir, parfois entre guillemets, bien soulignées, parfois au beau milieu de tout, sans distinction. Ces voix constituent les opposants d'une parole autre, celle du sujet féminin privé de représentations. Les noms de femmes rencontrées en cours de route n'installent pas un matriarcat, ne font pas figure de prêtresses, ne reçoivent pas idolâtrie. Elles sont plutôt là, singulières et solitaires, pour signaler des ruptures avec le pouvoir dominateur des affranchissements successifs qui constituent l'histoire non positivée du sujet. 
Meschonnic définit la conception hébraïque de l'histoire en opposition avec la conception grecque venue d'Hérodote (la chronique): "L'histoire se fait dans une succession infinie de naissances et de renaissances, Ce qui permet de concevoir les ruptures comme la continuité même de l'histoire." Dieu est lisible sous cet angle, sans téléologie.

Les citations et l'emprunt témoignent indéniablement de la présence de l'autre et du recours à l'autre. Il en va de même de la dédicace, avec certaines nuances: habituellement placée avant le texte proprement dit, la dédicace renvoie à une instance «réelle», c'est-à-dire fiasant partie de la vie privée de l'auteur. Ici, la dédicace advient dans le devenir du texte, à la p. 122, et elle s'adresse à un auteur déjà cité (François Charron)2: il n'y a pas d'extériorité au texte, pas de distinction entre le privé et le public, le discours ramasse tout. Le dédicataire prend dès lors position "actantielle», il collabore au «je viens» affirmé à la fin du texte.

Je veux maintenant pointer du doigt ce que Dieu nous révèle du sujet et de l'histoire (du sujet), et comment ce dire est indissociable de la pratique d'écriture, d'un travail lent et méandreux du langage. Un «je» s'affirme dans Dieu: quel est-il? Une voix émerge, à travers celle des autres. Par la critique du Nom, une femme cherche son prénom: à deux reprises elle croit l'avoir enten$\mathrm{du}$, mais il n'est pas temps de le revendiquer avec force, comme un moment de l'histoire: l'existence dans le prénom trouvera sa voie dans le prochain livre: ici, c'est l'effacement des images et l'entrée dans la parole.

Métamorphoses du Nom, mutations et mues de la voix, tout ce livre s'écrit dans le passage. Tous les mots dérivés de «passer» s'y retrouvent dans tous leurs sens: les pages 31 et 32 en donnent un bon exemple. On parle souvent de «double passage historique» que j'interprète comme le passage du sujet, à la fois individu et métaphore d'une réalité beaucoup plus grande que l'individu. Concernant l'énonciation, je veux insister sur le passage constant du «je» au «nous» et du «nous» au «je», entre lesquels il y a indécision, oscillation. Cela nous permettra de comprendre comment le «je» du texte parle un individu, Carole Massé, par exemple, mais n'est pas réductible à elle.

L'incipit nous lance sur la piste: "c'est une femme aux mille tours qu'il me faut dire aujourd'hui» (p. 9). D'abord un "elle», «une femme», associée dans les segments qui suivent au passé. "femme aux mille tours": habile et rusée magicienne, femme 
inépuisable, mais aussi femme fortifiée, entourée de «mille tours". Ce qui est dit au passé de la femme dans toute cette première page annonce aussi le projet du livre. Ensuite un «je», sous-entendu dans le «me", mais ce "je» n'énonce pas encore, c'est un «il» impersonnel qui le fait: «il me faut dire». Cela s'entend de deux manières: il faut dire «à moi», me raconter et il faut que je dise (ce "je» qui de son discours fera l'histoire). Le dire est associé à “aujourd'hui»: il y a dans Dieu, comme dans tout récit, un savoir, un "gnarus", mais ce savoir est doublé d'un non-savoir qui est le non-savoir du dire, de l'énoncer. D'où l'incertitude de la narration, ses transformations, son refus de former une totalité, un savoir absolu.

On ne peut vraiment comprendre le cheminement du «je» qu'en jetant un coup d'oeil sur les verbes qui l'accompagnent. Je me suis plu à noter tous ces passages où le "je" énonce; l'ensemble forme un beau poème, presque un texte en soi. J'en retranscris ici quelques-uns, dans l'ordre d'apparition. Cette entrée du «je» met d'ailleurs un certains temps à se produire, jusqu'à la page 16 on l'escamote de différentes manières. Enfin: je pars - je meurs (16); mais que sais-je - je sais - j'ignore (20); que n'ai-je été - je souffre (24); je avec tout le doute - je ne sais pas ce que je dis (25); imaginez-vous que je tue (31); c'est toute leur histoire d'amour bouchée par je (59); j'viens (72); moi je dis (75); j'entends là appeler - je vois un visage (82); je suis l'absente - je marche je ne sais pas ce que je dis et pourtant j'entends là appeler (100); je suis (104); je dis relire Dieu (105); c'est je qui meurt (115); j'viens (116); j'viens (118); je me couche - je veux voir (119); je suis vivante (120); j'écris - j'y fus - j'viens - j'y sois (121); je suis - je ne veux - j'ouvre - je vois - j'viens (122); je me tue à vous les répéter (124).

Un tel exercice est significatif en ce qu'il nous permet de retracer une progression et certaines constantes. Plus on avance, plus le «je» s'affirme, plus les verbes se font «décisifs». Le «je» émerge, non pas pour se définir, faire son portrait, s'identifier à des images, mais pour parler. La question "qui suis-je?", formulée dans l'émoi (p. 34), n'obtient aucune réponse positive: c'est une question qui se vide de sa substance et devient inutile quand le «je» se met à parler vraiment.

Souvent, le «je» se confond au «nous». À quoi ou à qui renvoie ce «nous»? II faut distinguer. 
Dans les segments «passerons-nous à la question préalable des rapports entre l'individu et l'État?» (p. 12) et «nous ne passerons plus sur les détails douloureux» (p. 14), le «nous» n'est pas associé à une communauté de personnes: c'est le «nous» du discours objectif, le «nous» impersonnel qui désencombre l'argumentation du «je» problématique. À la page 31, le «nous» adopte une autre couleur: «hélas! nous sommes percés de coups mortels nos bouches exhalent des odeurs de meurtre et derrière le théâtre la voix de l'homme sans lèvres à qui nos lèvres crient n'entre pas!" Le sujet "nous" dans cette phrase n'est pas exclusivement des femmes puisqu'il est suivi d'un verbe accordé au masculin; nous pouvons supposer qu'il enferme l'ensemble des êtres humains victimes de "l'homme sans lèvres"; pour vraiment comprendre, il faudrait rassembler tous les passages où ce «nous» revient, ainsi que «l'homme sans lèvres». Enfin, on peut repérer un «nous» des femmes-qui-écrivent: "on s'imaginait que nous écrivions comme des femmes mais nous écrivons comme des chattes» (p. 92), «nous tordons ainsi le cou aux mots d'auteur délivrées que nous sommes de cette paralysie prostitutionnelle où nous étions sans corps couvertes de lauriers pour camoufler le pourrissement» ( $p$. $112-113)$.

Voilà un «nous» beaucoup plus subtil que le traditionnel «nous les femmes» de nombreux discours féministes des années 1970. Si une solidarité s'établit, ce n'est plus sous le chef de la distinction biologique: solidarité bien piégée que celle qui suit pour critère l'unique représentation sexuelle. "d'ici nous nous écrivons maintenant sans nous nier elle et moi moi et lui lui et elle» (p. 114). Le féminin dans Dieu est un concept plus fondamental que la simple appartenance au sexe féminin. Le «je» se fond dans un "nous» qui n'est pas le «nous» des femmes en général. Ce «nous" renvoie aux femmes nommées, citées dans le livre: ces personnages féminins, ces Hélène, ces Ariane, ces Colette, ces peintres que sont Victoire et Artémisia Gentileschi, toutes ces femmes qui ont écrit l'histoire du sujet féminin et fissuré la Raison d'État. À ces femmes se joint le «je» du discours qui les rassemble, se joint Carole Massé écrivant Dieu, se joignent toutes celles qui écrivent, qui tentent d'écrire. Ce «nous», bien qu'il soit parfois marqué du féminin, laisse la porte ouverte à des désirs d'hommes: une phrase du genre «comment différencier nos désirs inconscients de leur conscient désir de nous penser repanser?" (p. 92) peut être endossée autant par un homme que par une femme. Dieu se laisse donc lire comme une critique du féminisme sans pour autant être une défense des hommes et de l'«humanité»: le discours de Dieu 
transporte la problématique sur d'autres plans, bousille l'idéologie; en cela, c'est une parole politique.

Voilà, une simple amorce d'interprétation de ce livre fourmillant, peu univoque. Plusieurs entrées donnent accès à Dieu, son processus de lecture est infini. Toutefois, il y a en ce roman un mythe, une insistance dans la voix, des éléments signifiants qui empêchent d'en dire n'importe quoi. Dieu est un livre difficile à récupérer, sans cesse installé dans la tension, radicalement subjectif et désirant. Livre de l'élucidation, d'une venue dans la mort et la perte, du oui consenti qui suit la négation du oui-oui initial, qui était aliéné. Sans se fixer sur une idéologie, corrosif en un sens, Dieu est en même temps une affirmation, un écrit performatif qui continue de se produire. La couverture du livre, malgré tout ce qu'elle concède au spectaculaire, rend compte du passage qui s'opère: coincée d'abord entre Dieu et la mère de Dieu, Carole Massé, ni mère ni vierge, peut enfin se montrer sans honte.

1. Une récente entrevue avec Carole Massé par Chantal Saint-Jarre («Écrire... après une analyse», Arcade, Montréal, no 16, octobre 1988, p. 89) contient cette précision: "J'avais déjà publié mon premier recueil de poèmes, Rejet, et rédigé le premier jet du roman que j'intiłulerais Dieu avant d'entreprendre mon analyse en 1975. 1978 est fort probablement l'année où le dernier jet se met en place. L'achevé d'imprimer est de mars 1979. (Note d'A.G.)

2. Cette dédicace («à François Charron sans qui ce texte ne serait pas ce qu'il devient") est un renvoi implicite à telle dédicace semblablement formulée ( "Pour Carole Hébert, sans qui ce texte ne serait pas ce qu'il devient.") dans Pirouette par hasard poésie de François Charron (coll. «Lecture en vélocipède", Montréal, Ed. de l'Aurore, 1975). Cela renvoie donc à l'année 1975. Voir note 1. 